\title{
TRABAJO, TEMPORALIDADES Y PROCESOS COLECTIVOS. TRANSFORMACIONES EN LAS SUBJETIVIDADES DE VENDEDORES AMBULANTES Y RECOLECTORES INFORMALES
}

Trabalho, temporalidade e processos coletivos. Transformações nas subjetividades de vendedores ambulantes e catadores informais

Work, temporalities and collective processes. Transformations in the subjectivities of street vendors and informal collectors

Travail, temporalités et les processus collectifs. Les transformations dans les subjectivités des vendeurs de rue et les collectionneurs informels

Mariano Perelman 1

Doctor en Antropología (UBA), licenciado en Antropología(UBA), Investigador Adjunto del Consejo Nacional de Investigaciones Científicas y técnicas (CONICET). Docente del Departamento de Antropología de la Facultad de Filosofía y Letras (UBA) y del Programa de Doctorado en Ciencias Sociales (UBA). Ha sido profesor invitado en diferentes universidades de Argentina y del exterior. Es autor de varios artículos en revistas nacionales e internacionales, ha compilado libros y escrito capítulos de libros sobre temas relativos a la desigualdad urbana, el trabajo precario y las pugnas porel uso del espacio público de los sectores populares.

\section{RESUMEN}

En este texto me propongo contribuir a los debates en relación a las formas colectivas de trabajo y de demanda a partir de la experiencia (histórica) de vendedores ambulantes y cartoneros que no han formado organizaciones. Antes que partir entonces de una supuesta obligatoriedad de acción o manera de pensar en función de una forma organizativa, de la reivindicación de derechos o modo de vida, un estudio atento a los procesos históricos argentinos, pero a la vez grupales pensando en las expectativas, campos de oportunidades y marcos de referencia nos permite comprender el modo en que las personas construyen las formas de ganarse la vida y el lugar que las relaciones interpersonales tienen en este proceso.

Palabras claves:Trabajo; vendedores ambulantes; cartoneros; procesos colecticos.

\section{RESUMO}

Neste texto pretendo contribuir para discussões sobre formas coletivas de trabalho e demanda a partir da experiência (histórica) dos vendedores ambulantes e catadores do lixo que não formaram organizações. Antes disso, a partir de uma suposta ação obrigatória ou pensar em termos de uma forma de organização de reivindicar direitos ou formas de vida, atentos ao estudo dos processos históricos da Argentina, pensar sobre expectativas,

1 mdp1980@yahoo.com.ar 
campos de oportunidades e marcos de referência nos permite compreender como as pessoas constroemformas de ganhar a vida e o lugar que as relações interpes soais têm neste processo.

Palavras chaves: Trabalho; vendedores de rua; catadores do lixo; processos coletivos.

\begin{abstract}
In this text I intend to contribute to the debates regarding collective forms of work and demand based on the (historical) experience of ambulant vendors and garbage collectors who have not formed organizations. Before starting from a supposed obligation of action or way of thinking in terms of an organizational form, of the claim of rights or forms of life, a study attentive to the Argentine historical processes, but at the same time grouping with expectations, fields of opportunity and frames of reference allow us to understand how people build the ways of making a living and the place that interpersonal relationships have in this process.
\end{abstract}

Key Words: Work; hawkers; cartoneros; collective processes.

\title{
RESUMÈ
}

Dans ce texte, j'ai l'intention de contribuer aux discussions en ce qui concerne les formes collectives de travail et de la demande á partir de l'expérience (historique) des vendeurs ambulants et des collectors des déchets qui n'ont pas formé des organisations. Avant cela d'une action obligatoire présumée ou de penser en termes d'une forme d'organisation des droits de réclamation ou les formes de vie, une étude centrée sur les processus historiques d'Argentine, mais aussigroupal attentif aux expectatifs, domaines d'opportunités et de cadres des références nous permet de comprendre comment les gens construisent des modes de vie et lieu que les relations interpersonnelles ont dans ce processus.

Mots clés: Travail ; les vendeurs ambulants; collectors des déchets; processus collectifs.

\section{INTRODUCCIÓN}

En Argentina los estudios en torno a los trabajadores organizados tienen una gran tradición (Acha y Quiroga, 2015; Suriano, 2006; Collado y Nieto, 2017). La lista es extensa. Por un lado, existe una fuerte historia sindical que se ha traducido en investigaciones. Por otro lado, las propias dinámicas del campo académico han centrado los estudios del trabajo en torno a formas específicas ligadas al empleo formal. Esta visión también ha signado los estudios pos-crisis de 2001 cuando el desempleo alanzó niveles alarmantes y comenzaron a surgir formas alternativas de trabajo y de demandar por el empleo (Perelman, 2007). Más cercano en el tiempo, con el crecimiento del empleo durante los gobiernos kirchneristas (20032015) los estudios en torno a las prácticas de los trabajadores organizados han ido teniendo nuevamente un auge. Si bien han existido estudios en torno a los trabajadores (Neiburg, 1988; Grimberg, 1997; Perelman, 2007), la Antropología del trabajo en
Argentina, y especialmente en Buenos Aires comenzó a consolidarse como campo en este contexto. Por un lado, un grupo más ligado al estudio de los trabajadores formales e industriales (Giniger, 2009; Guiamet, et. Al, 2017; NET, 2005; Palermo, 2012; 2016; Perelman, 2007; Soul, 2014; 2015). Por otro lado, una vez pasado el pico de la crisis neoliberal del 2001 y conforme se iban asentando formas de trabajo por fuera del mercado de trabajo se comenzaron a pensar las formas organizativas de los trabajadores de la economía popular (Fernández Álvarez, 2015; 2016) y los modos modos de ganarse la vida por fuera del mercado "formal de trabajo" (Perelman, 2007; 2011; 2017c).

La pregunta sobre por qué la gente se organiza tiene la misma importancia a la de por qué la gente no lo hace. Podríamos pensar que la "excepcionalidad" de alguna de las dos cuestiones -si es que la hubieradepende de los procesos sociales. Y allí entonces adquiere relevancia alguna de las preguntas. Además, ambas cuestiones (o las maneras de entender las demandas) resulta 
relevante porque abordar una de las dos formas implica -por oposición y por relación- pensar la otra de las formas. Y, a su vez, permite problematizar las nociones mismas en torno a la organización ¿Qué significa estar organizado? ¿Cómo se demanda por una mejora en las condiciones de vida?

En los últimos años he estado trabajando junto con personas que se dedican a la recolección informal de residuos y a la venta ambulante que no se organizan en movimientos o en sindicatos para demandar o generar mejores condiciones de vida.

Sin embargo, ello no implica que no se organicen, que no tengan sus normas, valores o formas de hacer política. En este texto me propongo contribuir a los debates en torno a las formas colectivas de trabajo y de demanda a partir de la experiencia (histórica) de vendedores y cartoneros que no han querido formar organizaciones. Este abordaje, al ser analizado en paralelo con las formas organizativas (ya sea a partir de experiencias concretas o desde los imaginarios y prácticas de los propios sujetos no organizados) posibilita ver las complejidades y heterogeneidades en el mundo popular y en relación al trabajo.

Antes que partir entonces de una supuesta obligatoriedad de acción o forma de pensar en función de una forma organizativa, de la reivindicación de derechos o formas de vida, un estudio atento a los procesos históricos argentinos, pero a la vez grupales pensando en las expectativas, campos de oportunidades y marcos de referencia nos permite comprender el modo en que las personas construyen las formas de ganarse la vida (de L'Estoile, 2014; Narotzky, 2016; Narotzky \& Besnier, 2014; Perelman, 2011a; 2017c) y el lugar que las relaciones interpersonales (ya sea que sean pensadas como formas organizativas o no) tienen en este proceso.

Mirando desde este lugar, resulta necesario no homogeneizar a los sujetos por la actividad a la que se dedican. Dicho de otra forma, categorías como las de "vendedor" o "recolector" homogeneiza prácticas heterogéneas, relaciones y actividades con diferentes niveles de aceptación social y personal. Creo que pensar la complejidad de las prácticas y de los modos de vida (en los que se incluyen las formas organizativas) debe basarse en los estudios etnográficos que den cuenta de esta diversidad.

Quizás sea necesario realizar una aclaración. Así como cuando se piensan los tiempos y espacios de trabajo, es necesario analizar los modos organizativos de forma similar. Así como muchas veces resulta difícil diferenciar entre los tiempos y los espacios de trabajo (¿cuándo termina el tiempo laboral?), existen una multiplicidad y yuxtaposición de organizaciones a las que las personas recurren cotidianamente. Por su parte, los sentidos que las personas otorgan a participar de espacios son diferentes y múltiples (ver por ejemplo Quirós, 2009) por lo que podríamos preguntarnos dónde termina la organización y su tiempo. Así como en los empleos formales, el tiempo y el espacio de trabajo suele exceder el "formal" (como llevarse trabajo a la casa), las prácticas en los diferentes espacios suelen ir más allá del "objetivo formal" de la organización.

Una buena forma de abordar estas complejidades es la de dejarse llevar por los actores. Ello implica reconstruir las lógicas nativas desde diferentes lógicas e intervenciones. Como ha marcado Renoldi (2015, pp. 419-420), en relación al movimiento de las personas que trabajan entre la legalidad y la ilegalidad en la triple frontera (argetina, brasileña y paraguaya), "las dinámicas locales evidencian que la población adopta diversos modos de organizarse para vivir, en los que combina actividades legales e ilegales, vínculos económicos, de parentesco y amistad, en redes transnacionales que hacen a la vida cotidiana local". Para Renoldi "el papel del Estado, a través de las instituciones que controlan las fronteras, es significativo, tanto por lo que prohíbe como por lo que propicia con sus prohibiciones, dando lugar 
a múltiples 'ilegalismos". Retomando la idea de Foucalut, entiende a los ilegalis mos como las prácticas que crean cierta autonomía en la tirantez explícita con los bienes jurídicos tutelados por la ley. Disputan así diferentes órdenes que pueden no responder a los modelos defendidos por el Estado de derecho $\mathrm{y}$, sin embargo, se sostienen en las tramas de la administración estatal, aunque no siempre exista la conciencia de que tales prácticas son punibles (pues pasan como favores, cuando alguien que debería reprimir facilita ciertas iniciativas, o simplemente como emprendimientos rentables) (Renoldi, 2015, p. 419).

En esta misma línea, pero utiliza ndo la noción de frontera en un sentido amplio de construcción de límites (como los simbólicos) (Bayón, 2013; Lamont \& Molnár, 2002; Saraví, 2015), es pensable para actividades que se desarrollan en el espacio público. Y para ello también es necesario tener en mente la precaución que marca Renoldi (2015: 420) cuando plantea que "comparto el principio de que deshacerse de los marcos analíticos que presuponen la existencia de errores que impiden alcanzar formas deseables de integración o desarrollo es posible adhiriendo al esfuerzo comprensivo a partir de abordajes etnográficos que contribuyan con la revisión de conceptos y paradigmas acerca del orden y del cambio". Para ello es necesario deshechar la calificación de prácticas en términos acusatorios o morales desde la perspectiva del "poder" y tomarlas como categorías analíticas.

Por ejemplo, y como desarrollaré, la venta ambulante y la recolección informal de residuos muestran la existencia de movilidades laterales (Kessler, 2012; Ruggiero \& South, 1997; Telles \& Hirata, 2010) entre diferentes tipos de trabajos (legales- ilegales). Un estudio descentrado de la visión del poder permite mostrar los modos en que los procesos reales son

2 Esta línea, no sólo es posible seguirla en los trabajos "informales" sino también en empleos más vividos por las personas. Más aún, permite complejizar los límites borrosos entre lo que es llamado legal- ilegal; formal- informal ${ }^{2}$.

Esto no implica negar la importancia de las presencias estatales (Manzano \& Ramos, 2015) y sus efectos en las subjetividades. Antes bien, es justamente antes de darlas por sentadas, ponerlas a dialogar en la construcción de los procesos sociales.

Centrarse en las experiencias, en las expectativas y en los marcos de referencia de las personas y de las presencias estatales permite comprender el modo en que todo ello tienen efectos en las formas organizativas $\mathrm{y}$ en las formas de establecerse en las tareas. A su vez, posibilita deconstruir modos hegemónicos de ver las actividades que se organizan por fuera de ciertos marcos organizativos y que suelen ser vistos como negativos (como decir que "son mafias").

Hechas estas aclaraciones, entonces, resta decir que el estudio de los modos de organizarse para vivir no puede ser pensado desde un deber ser. Como afirma Zenobi (2014) es necesario evitar las prenociones propias y prestar atención a lo que es relevante para los actores sociales. O lo que es relevante en la configuración social.

Es por ello que resulta necesario centrarse en qué significa para las personas ordinarias una vida digna a ser vivida, el modo en que justifican sus acciones, construyen argumentos, recuerdan el pasado en relación al presente (Perelman, 2010a; 2011a; 2015).

A partir de aquí, mostraré cómo el modo de vivir impacta en las formas en que las personas trabajan y se organizan para demandar u obtener mejores condiciones de vida. Estas mejores condiciones no pueden reducirse a la mera obtención de recursos materiales. El texto está dividido en dos grandes apartados. En el primero me referiré a la venta ambulante para luego

establecidos como en las imprentas en Colombia (Cfr. Velandia Diaz, 2017). 
centrarme en la recolección (informal) de residuos.

\section{La venta ambulante}

En los últimos años, la venta ambulante en Buenos Aires se ha transformado en un problema público ligado a la "ilegalidad" 3 . Si bien no hay datos oficiales sobre la cantidad de personas trabajando en las calles (los que hay son, por ejemplo, los de la Confederación Argentina de la Mediana Empresa, que sirven para básicamente- para denunciar la piratería y la ilegalidad de los vendedores ambulantes) existe un consenso de que el crecimiento ha sido notable. En los últimos años nuevos grupos sociales se han configurado como vendedores: vendedores de alimentos, manteros que venden cosas "truchas", ferias donde se mezclan productos nuevos, usados y robados, artesanos, migrantes de países africanos vendiendo bijouteria. También varios vendedores se han ido organizando en pos de demandar por mejores condiciones o para resistir los procesos expulsivos. Es posible que el ingreso a la actividad de personas con otras experiencias laborales -en las que la organización sindical ha sido importantehaya influido en el crecimiento de organizaciones de vendedores. Así también lo ha hecho las políticas de los gobiernos de Néstor (2003-2007) y Cristina Kirchner (2007-2015), las cuales han fomentado formas organizativas, así como la necesid ad de organizarse para demandar y acceder a planes estatales y de ONGs.

Mis estudios comenzaron en 2011 y se focalizaron con otros trabajadores: los vendedores ambulantes en trenes $\mathrm{y}$ colectivos. Y dentro de ellos en un grupo: los buscas.

Cualquier persona que viaje en los trenes de la ciudad reconocerá rápidamente

\footnotetext{
3 Pese a que ciertos grupos vienen sufriendo la persecusión policial desde hace varios años (ver los trabajos de Pita [2012a, 2012b]), con la asunción de Mauricio Macri como presidente de la Nación, la persecución de los vendedores creció notablemente
}

a personas vendiendo productos, músicos callejeros o personas pidiendo dinero. Más difícil le será reconocer entre ellos a los buscas ya que el modo de ganar dinero suele no diferir de otros vendedores. La distinción se produce a partir de una serie de prácticas $\mathrm{y}$ valores morales que hacen de la venta ambulante un modo de vida (2013a).

Si la venta ambulante se constituyó como un problema público ligado a la ilegalidad, los vendedores en trenes y colectivo no han sido objeto de las políticas -al menos masiva- de expulsión. Han quedado relativamente a salvo de las políticas expulsatorias implementadas para los que vendían en puestos callejeros.

Mi trabajo de campo se focalizó en dos líneas ferrocarril. Una que va hacia el norte de la ciudad y otra hacia el sur.

Por un lado, los medios de comunicación y ciertos sectores de la población asocian a los vendedores con la idea de "mafia". Con ello hacen alusión a una gran organización ilegal piramidal para la venta de productos en los transportes en el que los trabajadores serían explotados por alguien que les da mercadería para vender a cambio de una pequeña remuneración. La noción de mafia hace alusión también a prácticas violentas para no dejar que otros puedan trabajar en esos mismos espacios.

Esta visión puede ser reforzada por el uso de la violencia como herramienta legítima para dirimir conflictos o no permitir que nuevas personas se conviertan en vendedoras.

Quizás la paradoja en la que nos encontramos es que mientras la organización para algunos actores es el modo deseado, para otros es visto como la generación e instalación de prácticas ilegales en cadenas de obtención de dinero. Es posible que esta explicación forme parte de ciertos discursos en torno a la obtención de dinero de los sectores populares. Wilkis

y se transformó en una política de Estado. Así, durante el 2016 y en los primeros días del 2017, la Ciudad de Buenos Aires fue objeto de desalojos masivos de personas dedicadas a la venta callejera en la ciudad. 
(2013) da cuenta del discurso en torno a que el dinero dado por el Estado para los pobres es sujeto de reclamos colectivos ya que es percibido como "de todos". Es, de esta forma, moralmente reclamable por todos (léase aquí los sectores dominantes). Desde esta posición, el reclamo sobre el uso debido o indebido de aquel dinero es posible: sus usos legítimos estarían ligados a la supervivencia y, llegado el caso, a una escala de prioridades basados en los valores burgueses. Los sectores populares según esta visión no tendrían derecho -porque no saben- cómo usarlo/ gastarlo. La visión tutelar de los pobres ha sido recurrente a lo largo de la historia argentina (Alvarez Leguizamón, 2008). En esta misma línea, la organización de los sectores populares cuando no es por la "mera supervivencia" es cuestionada por ilegítima, o por su ilegalidad ${ }^{4}$. Esta posición remite, a mi entender, a una visión miserabilista que, como muestra Álvarez Leguizamón (2017), permea todos los niveles (incluso el de los investigadores 'progresistas'). Dice Álvarez Leguizamón, quien retoma la idea del anális is de Silvia Rivera Cusicanqui. (2017, p.106 nota al pie 41) "El miserabil is mo sería la creencia, la práctica y la retórica que reconoce las condiciones de miseria $y$ explotación de los sectores subalternos pero no su condición de sujetos históricos o de clases en lucha, lo que produce un efecto de anonimato colectivo; '...el miserabilismo (...)le permite a las clases dominantes la objetivación y subalterniazación de estas poblaciones, y la legitimación del clientelismo como nuevo modo de dominación anclado en redes escalonadas y verticales de manipulación y dominio. La noción de miseria, al igual que las más moderna de pobreza, despojan a los actores

\footnotetext{
4 Ver por ejemplo Auyero (2007) sobre los saqueos ocurrido en 2001 o Perelman (2017a) en el caso de tomas de tierras en el Parque indoamericano de 2011.

5 Ver Perelman 2017c

6 Las mutuales son entidades sin fines de lucro prestadoras de servicios, sostenidas por el aporte de sus asociados. Tienen bajo sus principios el de la solidaridad y ayuda mutua entre sus socios que se
}

populares (indígenas, mujeres, trabajadores) de su condición de sujetos de la historia' (2005: 171-172)". Por su parte, la visión neoliberal y de las élites sobre las movilizaciones populares en Argentina da cuenta de esta visión ${ }^{5}$.

Pero volviendo a los buscas, la mayoría de las personas con las que hice trabajo de campo no tenía intención de agruparse. Algunos de ellos han tenido experiencias organizativas, las más de ellas fallidas. Estos procesos han contribuido a la idea de que las cooperativas y a las mutuales $^{6}$ no funcionaban. Una tarde de 2014 Javier me comentó sobre lo ocurrido unos años atrás con una persona que había acercado la idea de unirse a una mutual: "Hubo un tiempo que uno de los que laburaba [trabajaba] acá se le ocurrió la idea armar una mutual para cuando nos enfermábamos o teníamos un accidente. Nosotros poníamos plata todos los meses y el tipo se rajó [escapó]con toda la guita [con todo el dinero juntado]". La experiencia organizativa trunca forjó imaginarios en torno a la organización de esta específica configuración social. "Ahora nosotros ayudamos a los que se enferman o necesitan algo. Cuando alguno está enfermo juntamos entre todo y le damos a la familia". Fernández Álvarez (2015) ha mostrado el modo en que las situaciones truncas en una cooperativa de recolectores permitieron ampliar los horizontes de lo posible para "sobreponerse a una vida marcada por la precariedad, la desigualdad, la falta de" (244). Si en ese caso las experiencias fallidas ampliaron el ámbito de posibilidad, en el caso de los vendedores fueron productivas en otro sentido: generaron una mayor seguridad en torno a que las formas organizativas no eran el mejor camino para

reúnen para solucionar problemas comunes, con la finalidad de contribuir al logro del bienestar material y espiritual de sus miembros. El vínculo está basado en la confianza y en la reciprocidad. Los socios de la mutualidad, llamados mutualistas, contribuyen a la financiación de la institución con una cuota periódica. Con el capital acumulado a través de las cuotas de los mutualistas, la institución brinda sus servicios a aquellos socios que los necesiten. 
una mejora en la calidad de vida. Al mismo tiempo, ello contribuyó a generar formas de solidaridad y afinidad cotidiana. Como desarrollé en otros trabajos (Perelman, 2013a; 2013b), son las obligaciones mutuas que se expresan en prácticas y valores morales cotidianos los que producen las fronteras de pertenencia a los grupos de vendedores. Ello implica la obligatoriedad de ciertos comportamientos para con los de adentro o los de afuera.

Estas formas organizativas remiten a la manera de entender el trabajo. La misma idea de busca -palabra que proviene de busca vidas- es comenzar todos los días solo con el dinero necesario para comparar mercadería para la venta. Los vendedores reivindican la falta de ataduras y la libertad de la tarea, el no "arreglar" con la policía. Todo ello es parte de un modo de vida que los buscas conocen bien.

Las trayectorias de los vendedores construyen marcos de referencias en torno a las formas de ganarse la vida y cómo conseguir mejoras en ellas. Muchos de los vendedores son personas que han tenido trabajos precarios o han vivido en la calle. El repertorio de actividades posibles ha generado marcos interpretativos con los que se lee la sociedad. Ello ha generado formas de diferenciarse con otros que produce solidaridades y diferencias dentro de los grupos de vendedores.

La noción de libertad y esta visión de busca vida no implica, entonces, la inexistencia de organización. Antes bien, las relaciones personales son necesarias para que la venta ambulante sea posible. Y estas relaciones se generan tanto al momento de venta arriba de los vagones o en los andenes, así como fuera de ellos.

Esta forma de comportarse que implica conductas obligatorias con otros actores y otros buscas es lo que para las personas de carne y hueso crea un valor diferencial en torno a los modos de vida. Son las prácticas las que generan valoraciones en torno a los modos de vida.

Los diferentes grupos sociales construyen modos de permitir los ingresos de nuevos vendedores, de fijar precios, de diferenciar espacios y momentos de venta. Así, los vendedores son parte de un colectivo que los protege $\mathrm{y}$ al que deben defender.

Como las experiencias en torno a la organización de la mutual generan marcos de referencia para prácticas en el presente, la idea de trabajo, en la que se inscribe esta noción de libertad en tanto noción nativa que remite a formas específicas de comprender la sujeción al empleo y al manejo de los tiempos, también permite comprender la falta de organización más formal de los vendedores.

Ser busca se construye cotidianamente a partir de formas de consumir, de pasar el tiempo, de comprar y vender mercadería. Y claro está, ser busca se contrapone a otras formas y grupos presentes en el espacio. Ser busca involucra a personas, moralidades, y diferentes regímenes de formalidades / informalidades, y de legalidades / ilegalidades sobre las cuales los vendedores no hacen diferenciaciones.

Algunas prácticas pueden ser consideradas -desde la legalidad- como "ilegales". Así, por ejemplo, el trabajo que realizan se da por fuera del control impositivo. A su vez, muchas prácticas son ilegalizadas o construidas como ilegales: entre ellas se destaca el "cierre del espacio de trabajo" que es visto como una forma de privatizar el espacio público para uso personal con fines "comerciales".

Todo ello, genera diferencias morales basadas en las experiencias y en las expectativas en torno a no sólo cómo conseguir dinero sino también a cómo vivir dignamente. Ello producía diferencias dentro del colectivo de vendedores. En el caso de los buscas, las valoraciones morales en torno a su forma de vida también llevan a valorar otras cosas más que el dinero, como pasar tiempo charlando con amigos, haciendo uso del tiempo libre $y$ manteniendo espacios de autonomía. De la misma forma, las condiciones de informalidad e ilegalidad de la actividad no 
son percibidas por los vendedores como un componente de incertidumbre ni visto como un problema, como una anormalidad. Antes bien, es parte de la configuración específica de la venta.

\section{La recolección}

Así como en el caso de la venta ambulante en la que es el modo de vida busca y las experiencias específicas las que han impactado en las formas organizativas, lo mismo ocurre en el caso de los recolectores de residuos caracterizados como informales (actividad conocida como cirujeo o cartoneo), pero de manera diferente. Si en el caso anterior es la reivindicación de un modo de vida de manera "positiva" lo que genera formas no organizativas, los recolectores durante varios años no se organizaron por la "negativa", o sea porque consideraban a la tarea como una actividad no deseada, lo que contribuía a la búsqueda de una salida antes que de una estabilización.

Cuando comencé a realizar trabajo de campo en 2002, la actividad estaba comenzando a crecer y a (re)organizarse. En términos colectivos, el nivel de organización de los cartoneros era bajo. Según los primeros datos existentes -que son de diciembre de 2003-, sólo el 1,9\% pertenecía a una cooperativa de recolectores y el $22 \%$ tenía algún plan social (muchos de ellos manejados por las organizaciones sociales). Según estos relevamientos, los recuperadores que querían formar parte de una cooperativa $(41,2 \%)$ eran casi la misma cantidad que los que no querían hacerlo $(41,3)^{7}$. Ello obviamente sin adentrarnos en

\footnotetext{
${ }^{7}$ Datos provistos por el PRU a partir de los registros realizados entre 2002 y 2003 en la ciudad.

${ }^{8}$ En otro trabajo (Perelman, 2010b) di cuenta de que algunas cooperativas de cartoneros funcionaban como modos en que los sectores populares accedían a toda una serie de estrategias no son exclusivas de esta forma organizativa. En este sentido, me alejo de los que analizan las prácticas de los sujetos en función de la ideología cooperativa y los juzgan por su cercanía o lejanía con estos ideales. Por el contrario, me interesa mostrar que en las relaciones
}

qué significaba una cooperativa para los recolectores y el modo en que las políticas de estado generan modos organizativos ${ }^{8}$. La experiencia de la recolección en el contexto del gobierno kirchnerista trajo consigo nuevos aires y las organizaciones sociales ganaron terreno y legitimidad social. Las cooperativas de recolectores -como parte de una política de Estado tanto del macrismo en la ciudad $^{9}$ como del kirchnerismo a nivel nacional- fueron creciendo y se fueron organizando en movimientos y confederaciones de trabajo. Hoy no existen datos oficiales sobre la cantidad de recolectores existentes ni los que están organizados, pero el nivel organizativo relacionando a la actividad es importante ${ }^{10}$.

He de marcar aquí una importante diferencia entre los vendedores y los recolectores. En primer lugar, si la venta ambulante como problema público se inscribió en la línea de la ilegalidad, el de la recolección informal, lo hizo en el cuidado del medio ambiente. Así, el propio gobierno fomentó la organización en cooperativas de trabajo que funcionaron tanto como una forma de mejorar las muy precarias condiciones de vida de los recolectores, así como de condenarlas a ellas. Las cooperativas de cartoneros son efectos de Estado. Y digo condenarlas porque, a diferencia del caso de los vendedores, el cirujeo ha sido para muchos un camino no buscado que se ha ido naturalizando.

El crecimiento de la actividad que se dio como parte de la desarticulación del mercado de trabajo desde las políticas neoliberales, hizo que miles de personas acostumbrados a ganarse la vida a partir de

hay un algo más. Creo que son modos y prácticas que están instituidas y forman parte de las redes a las que los cirujas apelan para poder sobrevivir.

9 Mauricio Macri, antes de ser presidente de la República, fue Jefe de Gobierno de la Ciudad de Buenos Aires por dos períodos consecutivos (20072015).

${ }^{10}$ Un relevamiento de los actuales estudios sobre el tema da cuenta también de la creciente importancia que tienen las cooperativas. 
empleos formales, informales y changas $^{11}$, recurran a la búsqueda en la basura de materiales vendibles. Para la mayoría de ellos, el cirujeo no había sido una actividad "deseada". No era considerada un modo digno de ganarse la vida. $\mathrm{Si}$ bien comenzaron a organizarse en pos de demandas concretas - poder acceder a la ciudad en trenes, tener campañas de vacunación o que el estado les provea de ciertos medios para mejorar las condiciones- la idea de que era una actividad pasajera generó, sobre todo en los primeros años de mi trabajo de campo, una reticencia a cualquier tipo de modo organizativo ya que la actividad era vista como una cuestión pasajera.

Aquí las condiciones de vida actuales reales y los marcos de referencia, así como las expectativas de vida futura funcionaron como antídotos ante la posibilidad de ciertas formas de organización. La naturalización del cirujeo como modo (legítimo) de vida, se fue dando conforme se iba generalizando la actividad en los barrios donde los cartoneros vivían. Durante 2002, barrios enteros vivían de la recolección de residuos. El cartoneo era una posibilidad concreta. Pese a ello, las transmisiones generacionales seguían girando en dirección al mercado de trabajo como modo legítimo de ganarse la vida. Los tiempos de crisis son constitutivos de espacios y temporalidades que son simultáneamente compartidas y distintivas (Goddard, 2017). Los cambios, efectos de un largo proceso de reformas estatales neoliberales explican, en algún sentido, "las diferentes condiciones de vida que se encuentran en las generaciones sucesivas y que encuentran sus expresiones en las diferentes condiciones para generar continuidades y rupturas afectando la transmisión generacional" (Goddard, 2017, p. 7, traducción propia). Esta transmición es de conocimientos así como de valores morales. "No quiero esto para mis hijos. Quiero que consiga un trabajo y haga algo

11 Las changas son trabajos ocasionales. Pueden hacerse en paralelo a otro trabajo. digno" me dijo una vez Esteban, un cartonero que cuando nos conocimos hacía menos de un año que recolectaba. El intento de proteger a los jóvenes de la realización del cirujeo, el intento de transmisión generacional de los valores ligados al "trabajo" y no al "cartoneo" -recurrente por cierto en diferentes personas con las que hice trabajo de campo- también da cuenta del modo en que, en los momentos de crisis, ciertas actividades son posibles para unas generaciones, pero se busca resguardar a las siguientes de ella.

Durante mi trabajo de campo podía apreciar que las prácticas cotidianas daban cuenta de estar cartoneando, pero no de ser cartonero. Esta diferencia no es menor en la constitución de modos de ganarse la vida que dan cuenta de los entramados relacionales, las formas de entender el pasado y pensar el futuro en relación a la recolección.

Mi trabajo de campo también dio cuenta de este complejo equilibrio entre la necesidad de ganarse la vida y la intención de salir de ella. Por un lado, los comportamientos con los vecinos giraban en torno a la necesidad de conseguir material para vender o usar, pero también la de mostrar las capacidades para poder conseguir "un trabajo" (2011b).

A diferencia del caso de la venta ambulante, en el del ciruejo eran las condiciones pasadas y puestas en perspectiva las que generaban modos (no) organizativos de reconocimiento de derechos a partir del cirujeo. Las trayectorias de vida de varios de los recolectores reconocen formas sindicales o partidarias de organización social y/ política. Sin embargo, la sensación de estar haciendo algo que no querían generó, en gran parte de los recolectores, un rechazo, sobre todo en los primeros años de trabajo, de cualquier forma, que implicase una estabilización en la actividad.

La idea de autonomía y libertad que aparecían como valores morales positivos

\footnotetext{
"hice changas en el almacén hasta que encontré un buen trabajo".
} 
para los que realizaban la tarea (para contraponer a experiencias previas nuevamente aquí se pueden apreciar los marcos de referencia) también signaron formas de no organizarse colectivamente. Así, ante la pérdida del empleo, una de las formas en que los cartoneros comenzaron a reivindicar la tarea que realizaban era la libertad. Moverse por la ciudad, no tener horarios, no depender de jefes eran argumentos expresados como formas reivindicativas para trabajar "solos". Estas visiones impactaban en la falta de intención de trabajar con otros de formar solidaria $y$ cooperativa de trabajo.

Al igual que en la venta ambulante las relaciones interpersonales son centrales en la constitución del mercado de recolección. Las interacciones cotidianas entre los recolectores y entre ellos y los vecinos, los clientes y los depositeros ${ }^{12}$ generan obligaciones recíprocas que van posibilitando modos de actuar, y configurando recorridos específicos.

Las relaciones legales/ ilegales también constituyen aquí límites borrosos que los actores traspasan sin problemas. Al igual que en el caso de la venta ambulante, es un trabajo informal. La actividad hasta 2003 estaba prohibida y ello generó reacciones del por entonces candidato a Jefe de Gobierno y actual presidente de la Nación Mauricio Macri. En un reportaje que dio al Diario La Nación publicada el 27 de agosto de 2002 puede leerse ${ }^{13}$ :

-Uno de los temas que más preocupan a los porteños es el de los cartoneros. ¿Qué propone usted al respecto? -Un nuevo diseño ambiental. Hay crisis en el manejo final de los residuos y el cirujeo es

\footnotetext{
12 Vecinos da cuenta de las personas que viven en la zona de recolección. Clientes por su parte remite a las personas que les guardan los residuos. Depositeros es utilizado para referir a las personas que trabajan en los depósitos de compra y venta de materiales reciclables a los que los cartoneros venden lo recolectado.
}

un descontrol absoluto. Formar cooperativas no resuelve nada. Este es un negocio millonario y los cartoneros tienen una actitud delictiva porque se roban la basura. Además, no pagan impuestos y la tarea que realizan es inhumana. En otras sociedades, el tratamiento de la basura se hace en lugares cerrados, con elementos, con gente contratada ad hoc. (...) no pueden estar en la calle. Los vamos a sacar de la calle. -¿Cómo?

-Ejerciendo la ley. Están cometiendo un delito. Tenés que darles una alternativa, como contratar a unos miles para que hagan la separación de residuos dentro de los centros de procesamiento, y no en la calle.

- ¿Y al que siga en la calle? -Me lo llevo preso. Vos no podés alterar el orden en algo que es un delito, porque es tan delito robar la basura como robarle a un señor en la esquina.

Las detenciones policiales -por su parte- también han sido recurrentes. Éstas, sin embargo, no son explicables por esta ilegalidad sino por ser pobres circulando por la ciudad. En los recolectores, no está presente esta visión de ilegalidad de sus prácticas. Antes bien, recurren a argumentos y elecciones morales relacionados con formas legítimas de ganarse la vida.

13 "A Los Recolectores Informales De Basura Los Vamos A Sacar De La Calle", Diario La Nación Del 27 De Agosto De 2002. Disponible On Line http://www.lanacion.com.ar/425929-a-losrecolectores-informales-de-basura-los-vamos-a$\underline{\text { sacar-de-la-calle }}$ 
Zenobi (2014: 45) dice que "[d]esde el punto de vista de los actores, al momento de evaluar una conducta determinada resulta relevante considerar tanto los juicios emitidos por quien lleva adelante una acción específica, así como las valoracio nes sostenidas por otras personas que forman parte de la misma configuración". El trabajo "individual" o familiar era para muchos valorado como una forma de -pese a estar haciendo algo no deseado- recuperarse y "ganarse la vida dignamente". Esta forma de trabajo era contrapuesta a la de otros cartoneros que trabajaban recolectando para otros de forma "organizada" constituía modos de diferenciar las prácticas en términos morales (de legitimidad) antes que de (i)legalidad. Los modos en que las personas de carne y hueso viven los procesos y construyen formas relacionales de pensar las tareas genera maneras de comprender las actividades más allá, pero en relación con otros discursos. Es en este sentido que es posible comprender los modos en que los recolectores piensan los modos organizativos.

\section{A modo de cierre. Temporalidades y experiencias colectivas en el trabajo callejero}

En este trabajo he querido mostrar el modo en que los espacios de referencia y los horizontes de expectativas posibilitan comprender los imaginarios en torno al pasado, al presente y al futuro. En relación a los modos de ganarse la vida de L'Estoile (2014, p. s64 traducción propia) plantea que "Estos marcos de referencia (que incorporan la experiencia individual y colectiva) definen tanto las formas en que se experimenta e interpreta el mundo como las expectativas respecto del futuro. $\mathrm{La}$ combinación de un campo de oportunidades dado $y$ un marco específico define 'horizontes de expectativa' individuales y colectivos. Estas nociones son, por supuesto, reflexivas: se aplican no solo a aquellos que deseamos comprender sino a nuestra propia 'forma de vida' ". Los modos en que el pasado, el presente y el futuro son vividos pueden contribuir a generar formas asociativas específicas para demandar por mejoras o puede constreñir su formación. Los casos que traje dan cuenta de estas diferencias.

Un estudio atento a las especificidades históricas y sociales de las configuraciones sociales permite comprender los modos y los argumentos en torno a lo que es una vida digna van configurando interrelaciones que adquieren diferentes sentidos. Así, mientras que en el caso de la venta ambulante el modo de vida deseado implica una libertad en la que las formas organizativas irían en detrimento de ella; en el caso de la recolección informal es el modo de vida no deseado el que impide la organización.

Por otro lado, he mostrado que la misma noción de organización para demandar debe ser analizada etnográficamente. Si bien en ambos casos las personas hablan de la libertad, mis observaciones dan cuenta de fuerte entramado organizacional que permite a vendedores y recolectores generar las condiciones de demandas en torno a su actividad.

Me gustaría concluir el presente texto marcando que los cambios sociopolíticos argentinos, así como la naturalización de cierta actividad como un modo legítimo de ganarse la vida también han impactado en los modos organizativos. Las diferentes temporalidades de los procesos sociales son centrales en los modos en que las personas calculan lo que es una vida digna y transforman los marcos de referencia y las condiciones de posibilidad de acción.

En el caso del cirujeo, por ejemplo, la instalación de la actividad en relación al medio ambiente, la naturalización de la tarea como una forma posible de acceder a recursos y el fomento estatal de la cooperativa como un modo organizativo ha ido generando modos organizativos que han contribuido a la mejora en las condiciones de trabajo (objetivas y subjetivas) de los 
recolectores. En el caso de la venta ambulante, ante la creciente persecución y privatización de los espacios públicos han también comenzado a pensar en modos de organizarse en pos de demandar por su modo de vida.

En todo caso, todo ello muestra que los marcos de referencia ni los horizontes de expectativa son estancos; que los modos de entender el futuro, el presente y el pasado van cambiando. Un estudio atento a las temporalidades de los procesos sociales permite comprender los cambios en los múltiples cálculos que las personas de carne $\mathrm{y}$ hueso hacen en pos de vivir una vida digna.

\section{Referências}

Acha, O. \& Quiroga N. (comps.) (2015) Asociaciones y política en la Argentina del siglo veite. Entre prácticas y expectativas. Buenos Aires: Prometeo.

Álvarez Leguizamón, S. (2008). Pobreza y Desarrollo en América Latina. Salta: Universidad Nacional de Salta.

Álvarez Leguizamón, S. (2017). Formas de racismo indio en la Argentina $y$ configuraciones sociales de poder. Rosario: Prohistoria.

Auyero, J. (2007). La moralidad de la violencia popular: el caso de los saqueos populares de diciembre de 2001. En A. R. Isla (Ed.), En los márgenes de la ley: inseguridad y violencia en el Cono Sur (pp. 47-68). Buenos Aires; Barcelona; México: Paidós.

Bayón, M. C. (2013). Hacia una sociología de la pobreza: la relevancia de las dimensiones culturales. Estudios sociológicos, XXXI (91), 87-112.

Collado, P. \& Nieto, A. (2017) Sindicalismo, sindicatos, movimientos, acciones y organizacionesisépide los trabajadores. P. Collado; J. L. Bonifacio; G. Vommaro (coords.), Estudios sobre ciudadanía, movilización y conflicto social en la Argentina contemporánea (pp.45-130). Buenos Aires: CLACSO y PISAC.

de L'Estoile, B. (2014). «Money Is Good, but a Friend Is Better»: Uncertainty, Orientation to the Future, and «the Economy». Current Anthropology, 55(S9), S62-S73.

Fernández Álvarez, M. I. (2016). Experiencias de precariedad, creación de derechos y producción colectiva de bienestar(es) desde la economía popular. Ensamble, 3 (3/4), 72-89.

Fernández Álvarez, M. I. (2015). La potencialidad de las situaciones truncas en el estudio de la política colectiva. En M. I. Fernández Álvarez (Ed.), Hacer juntos(as). Dinámicas, contornos y relieves de la política colectiva (pp. 223-244). Buenos Aires: Biblos.

Giniger, N. (2009). Industrial Anthropolo gy in Argentina. Anthropology Work Review, 30, 10-13.

Goddard, V. A. (2017). Work and Livehoods: An Introduction. En S. Narotzky \& V. A. Goddard (Eds.), Work and livelihoods: history, ethnography and models in times of crisis (First published, pp. 1-27). New York, NY London: Routledge.

Guiamet, J.; G. Philipp; S. Vitali \& v. Vogelmann (2017) La antropología del trabajo en Rosario (Argentina) y su región: desafíos, rupturas y continuidades. Revista Latinoamericana de Antropología del trabajo, 1, 1-26. 
Grimberg, M. (1997) Demanda, negociación y salud. Antropología social de las representaciones y prácticas de los trabajadores gráfico 1984-1990. Buenos Aires: CBCUBA.

Kessler, G. (2012). Movilidades laterales, delito, cuestión social y experiencia urbana en las perisferias de Buenos Aires. Revista de Ciencias Sociales, 25(31), 120-142.

Lamont, M., \& Molnár, V. (2002). The Study of Boundaries in the Social Sciences. Annual Review of Sociology, 28(1), 167-195.

Manzano, V., \& Ramos, A. (2015). Introducción. Procesos de movilización y de demandas colectivas: estudios y modos de abordar 'lo político' en la vida social. Identidades, 8(5), 1-25.

Narotzky, S. (2016). Between Inequality and Injustice: Dignity as a Motive for Mobilization During the Crisis. History and Anthropology, 27(1), 7492.

Narotzky, S., \& Besnier, N. (2014). Crisis, Value, and Hope: Rethinking the Economy: An Introduction to Supplement 9. Current Anthropology, 55(S9), S4-S16.

Neiburg, F. (1988) Fábrica y Villa Obrera. Buenos Aires: CEAL.

NET. (2005). ¿Por qué una Antropología del Trabajo? Aportes para la discusión de su pertinencia disciplinar. En Actas del $1^{\circ}$ Congreso Latinoamericano de Antropología. Versión digital.

Palermo, H. (2013). Cadenas de oro negro en el esplandor y ocaso de YPF. Buenos Aires: Antropofagia.
Palermo, H. (2017). La producción de la masculinidad en el trabajo petrolero. Buenos Aires: Biblos.

Perelman, M. D. (2007). Theorizing Unemployment: Toward an Argentine Anthropology of Work. Anthropology of Work Review, 28(1), 8-13.

Perelman, M. D. (2010a). Memórias de la quema. O cirujeo em Buenos Aires trinta anos depois. Mana, 16(2), 375399.

Perelman, M. D. (2010b). Repensando el cirujeo a partir de una cooperativa de cartoneros de la Ciudad de Buenos Aires. Revista Argentina de Sociología, 8(14), 177-197.

Perelman, M. D. (2011a). La construcción de la idea de trabajo digno en los cirujas de la ciudad de Buenos Aires. Intersecciones en antropología, 12(1), 69-81.

Perelman, M. D. (2011b). La estabilización en el cirujeo de la ciudad de Buenos Aires. Una aproximación desde la antropología. Desarrollo económico, 51(201), 35-57.

Perelman, M. D. (2013a). Trabajar en los trenes. La venta ambulante en la ciudad de Buenos Aires. Horizontes Antropológicos, 19(39), 179-204.

Perelman, M. D. (2013b). Trabajar, Pedir, Vender. El Caso de los Vendedores Ambulantes en Trenes de la Ciudad de Buenos Aires, Argentina. The Journal of Latin American and Caribbean Anthropology, 18(2), 231250.

Perelman, M. D. (2015). Viejos y nuevos cirujas. Construcciones temporales $\mathrm{y}$ espaciales en la Ciudad de Buenos 
Aires. Cuadernos de antropología social, 42, 125-141.

Perelman, M. D. (2017a). Construyendo la legitimidad. Esperas y argumentos morales en la toma del Parque Indoamericano de la Ciudad de Buenos Aires. Dilemas - Revista de Estudos de Conflito e Controle Social, 10 (2), 241-258.

Perelman, M. D. (2017b) Notes about Racist Argentina and a Class-based Government.

American Anthropologist, 119 (3), 532-534

Perelman, M. D. (2017c). Pensando la desigualdad urbana desde el trabajo callejero. En M. D. Perelman \& M. Boy (Eds.), Fronteras en la ciudad:(Re)producción de desigualdades y conflictos (pp. 1944). Buenos Aires: Teseo.

Pita, M. V. (2012a). Mitologías porteñas en torno al poder policial. Policía, contravenciones y gestión de ilegalismos en la Ciudad de Buenos Aires. La Biblioteca, 12, 182-209.

Pita, M. V. (2012b). Poder de polícia e administração de grupos sociais: o caso dos vendedores ambulantes senegaleses na Cidade Autônoma de Buenos Aires. En V. da S. Telles, C. Azaïs, \& G. Kessler (Eds.), Ilegalismos, cidade e política (pp. 109-140). Belo Horizonte: Fino Traco.

Quirós, J. (2009). Ser piquetero, estar con los piqueteros. Hacia una etnografía descentrada de los movimientos como objeto de análisis. En M. Grimberg, M. I. Fernández Álvarez, \& M. C. Rosa (Eds.), Estado y movimientos sociales: estudios etnográficos en Argentina y Brasil (1. ed, pp. 37-52). Buenos Aires: Antropofagia y Facultad de Filosofía y Letras.
Renoldi, B. (2015). Estados posibles: travesías, ilegalismos y controles en la Triple Frontera. Etnográfica. Revista do Centro em Rede de Investigação em Antropologia, vol. 19 (3), 417-440.

Ruggiero, V., \& South, N. (1997). The LateModern City as a Bazaar: Drug Markets, Illegal Enterprise and the «Barricades». The British Journal of Sociology, 48(1), 54-70.

Saraví, G. A. (2015). Juventudes fragmentadas: socialización, clase y cultura en la construcción de la desigualdad (Primera edición). México, D.F: FLACSO México: CIESAS.

Soul, J. (2014) Somiseros: conformación y devenir de un colectivo obrero en una perspectiva antropológica. Rosario: Prohistoria.

Soul, J. (2015) La Antropología del Trabajo contemporánea Una revisión histórica de la constitución de su campo disciplinar. Revista de la Escuela de Antropología, XXX, $67-84$.

Suriano, J. Los dilemas actuales de la historia de los trabajadores. En J. Gelman (comp.) La historia económica argentina em la encrucijada (pp. 285-306). Buenos Aires: Prometeo.

Telles, V. da S., \& Hirata, D. (2010). Ilegalismos e jogos de poder em São Paulo. Tempo Social, 22(2), 39-59.

Velandia Diaz, D. Antropología del trabajo e historia social. El mundo del trabajo en las imprentas de Colombia. Revista latinoamericana de antropología del trabajo 2 (segundo semestre) en prensa. 
Wilkis, A. (2013). Las sospechas del dinero. Moral y economía en la vida popular. Buenos Aires: Paidós.

Zenobi, D. (2014). Familia, política y emociones. Las víctimas de Cromañón entre el moviemiento y el Estado. Buenos Aires: Antropofagia.

Data de submissão: 06/07/2017

Data de aceite: 28/10/2017 